\title{
Financial Research Support for Ecotoxicology and Environmental Chemistry in Germany - Results of an Online Survey
}

\section{Fördersituation ökotoxikologischer und umweltchemischer Forschung in Deutschland - Ergebnisse einer Online-Befragung}

Henner Hollert*1, Juliane Filser², Roger Häußling ${ }^{3}$, Michaela Hein, Michael Matthies ${ }^{5}$, Jörg Oehlmann ${ }^{6}$, Hans-Toni Ratte Martina Roß-Nickoll7, Andreas Schäffer', Martin Scheringer ${ }^{8}$ and Andreas Schiwy'

\begin{abstract}
In recent years several initiatives addressed the inadequate financial support of pollutant-related environmental research in the Federal Republic of Germany. For an objective analysis about the research funding in ecotoxicology and environmental chemistry in Germany, an anonymous online survey was prepared. With support of the Society of Environmental Toxicology and Chemistry (SETAC) - German Language Branch and the German Chemical Society (GDCh) - Division of Environmental Chemistry and Ecotoxicology an invitation to participate in the survey was sent to all members of these two major associations for ecotoxicology and environmental chemistry in Germany (D), Switzerland (CH) and Austria (A). Only senior staff from the areas academics, government and industry was invited. The present article introduces the results of the survey. It is segmented in a section on socio-economic characterization of the participants, a section on support of research by the DFG and a section on funding by other funding organizations. A total of 71 male and female scientists in senior positions from various areas participated in the survey. The results revealed that the participants are to be classified as having excellent records. $48.5 \%$ of the respondents had submitted at least one research proposal to the DFG in the past, but one third actually received financial support by the DFG. 64\% are not satisfied with the DFG support of pollutantrelated research, only $7 \%$ are satisfied. It turned out that the research proposals are generally very heterogeneous and thus distributed to various units of the DFG with geosciences, water research and chemistry ranking highest, followed by biology and ecology. $91.2 \%$ of the respondents indicated that they have submitted proposals for research funding to other funding institutions (except the DFG), and $83.6 \%$ already have received appropriate external funding. $62.3 \%$ of the scientists believe that overall support for chemicals-related research in recent years has significantly deteriorated or is deteriorating. The vast majority of respondents (60.9\%) is dissatisfied with the funding situation, only $10.9 \%$ are satisfied. When asked "Is the total research funding in other European countries superior to that in Germany?" $30 \%$ answered "yes", $9 \%$ answered "no" and $61 \%$ "do not know". In summary the survey shows that the funding situation for research in ecotoxicology and environmental chemistry in Germany is improvable, and with respect to the DFG it is considered problematic. The noticeable under-representation of the DFG in comparison to other funding institutions shows that the most important funding body in Germany does not take into account the requirements of pollutant-related environmental sciences. In particular the answers to the open questions regarding improvement of research funding should be used as a basis for an open dialogue of chemicals-related environmental research institutes with the funding institutions DFG, BMBF and DBU and corresponding institutions in $\mathrm{CH}$ and $\mathrm{A}$.
\end{abstract}

Keywords: Ecotoxicology, Environmental Chemistry, Online Survey, Research funding

*Correspondence: henner.hollert@bio5.rwth-aachen.de

'Department of Ecosystem Analysis, Institute for Environmental Research (Biology V), RWTH Aachen University, Worringerweg 1, 52074 Aachen, Germany Full list of author information is available at the end of the article

\section{SpringerOpen ${ }^{\circ}$}

(c) 2011 Hollert et al; licensee Springer. This is an open access article distributed under the terms of the Creative Commons Attribution License (http://creativecommons.org/licenses/by/2.0), which permits unrestricted use, distribution, and reproduction in any medium, provided the original work is properly cited. 


\section{Zusammenfassung}

In den vergangenen Jahren gab es verschiedene Initiativen, die auf die unzureichende Fördersituation der Schadstoffbezogenen Umweltwissenschaften in der Bundesrepublik Deutschland aufmerksam gemacht haben. Um eine objektive Analyse über die Fördersituation der Ökotoxikologie und Umweltchemie in Deutschland zu erhalten, wurde eine anonyme Online-Befragung ausgearbeitet. Mit Unterstützung der Society of Environmental Toxicology and Chemistry (SETAC) - German Language Branch und der Gesellschaft Deutscher Chemiker (GDCh) - Fachgruppe für Umweltchemie und Ökotoxikologie wurde eine Einladung zur Teilnahme an der Befragung an alle Mitglieder dieser beiden maßgeblichen Verbände der Ökotoxikologie und Umweltchemie im deutschsprachigen Raum versendet. Nur leitende Mitarbeiter aus den Bereichen Forschung, Behörden und Industrie sollten an der Befragung teilnehmen. Die Befragung gliedert sich in eine Sektion zur sozioökonomischen Charakterisierung der Teilnehmer, eine zur Förderung der Forschung durch die DFG und eine zur Förderung durch andere Geldgeber. Insgesamt haben 71 Wissenschaftler und Wissenschaftlerinnen in leitenden Positionen aus verschiedenen Sparten an der Befragung teilgenommen. Die Ergebnisse zeigen, dass die Teilnehmer als sehr leistungsstark eingestuft werden können. 48,5\% der Befragten hatten bereits einen Antrag bei der DFG gestellt. Ein Drittel der Befragten gaben an, eine Förderung durch die DFG erhalten zu haben. 64 \% sind mit der Förderung Schadstoffbezogener Umweltwissenschaften durch die DFG nicht zufrieden, nur $7 \%$ sind zufrieden. Es zeigte sich, dass die Anträge insgesamt sehr heterogen auf verschiedene Fachbereiche der DFG verteilt sind. Geowissenschaften, Wasserforschung und Chemie nehmen die ersten Ränge ein, vor Biologie und Ökologie. Im Gegensatz dazu gaben 91,2\% der Befragten an, dass Sie bereits Drittmittelanträge bei anderen Förderinstitutionen (außer der DFG) gestellt haben, und 83,6 \% wurden bereits entsprechende Drittmittelanträge bewilligt. 62,3\% der Befragten sind der Meinung, dass sich die Fördersituation für die Schadstoffbezogenen Umweltwissenschaften in den letzten Jahren insgesamt verschlechtert oder sogar deutlich verschlechtert hat. Der überwiegende Anteil der Befragten (60,9\%) ist mit der Fördersituation durch Drittmittelgeber unzufrieden, nur $10,9 \%$ sind damit zufrieden. Auf die Frage „Ist die Forschungsförderung im europäischen Ausland insgesamt besser als in Deutschland?" antworteten $30 \%$ mit "ja", 9 \% mit „nein" und 61 \% mit "ich weiß nicht". Zusammenfassend ergab die Befragung, dass die Fördersituation der Ökotoxikologie und Umweltchemie in Deutschland insgesamt als steigerungsbedürftig, bei der DFG jedoch als problematisch zu bewerten ist. Die auffällige Unterrepräsentation der DFG im Vergleich zu anderen Drittmittelgebern verdeutlicht, dass die wichtigste Förderinstitution Deutschlands den Bedürfnissen der Schadstoffbezogenen Umweltwissenschaften nicht hinreichend Rechnung trägt. Insbesondere die Antworten auf die offenen Fragen bezüglich Verbesserungsmöglichkeiten der Forschungsförderung sollten als Grundlage für einen offenen Dialog der Schadstoffbezogenen Umweltforschung mit den Drittmittelgebern DFG, $\mathrm{BMBF}$ und DBU bzw. den entsprechenden Institutionen in $\mathrm{CH}$ und $\mathrm{A}$ genutzt werden.

\section{Einführung und Hintergrund}

In den vergangenen Jahren haben verschiedene Initiativen auf die unzureichende Fördersituation der Schadstoffbezogenen Umweltwissenschaften in der Bundesrepublik Deutschland aufmerksam gemacht.

In diesem Kontext wurde UFoH, ein Verbund umweltforschender Hochschulinstitute gegründet, dem über 70 leitende Mitarbeiter von Hochschulinstituten angehören und der sich an Hochschulinstitute und -arbeitsgruppen richtet, die sich in Forschung und Lehre mit dem Verhalten und den Auswirkungen bisher nicht oder unzureichend erforschter Stoffe in der Umwelt befassen. Ziel der Initiative ist es, die Bedeutung der stoffbezogenen Umweltwissenschaften und ihrer Verankerung an Universitäten herauszustellen. Von den Initiatoren dieser Initiative wurde ein Positionspapier [1] publiziert, welches die Verdienste und auch die Bedürfnisse einer Chemikalien-bezogenen Umweltforschung darstellt. Durch stetig zunehmenden Verbrauch von Chemikalien in allen Lebensbereichen mit Emissionen in alle Umweltmedien ist dieses Forschungsgebiet für die Gesellschaft und Umweltqualität von höchstem Interesse. Um auch zukünftig diese Aufgabe erfüllen zu können, besteht ein hoher Bedarf an exzellenter Forschung und Ausbildung. Wichtige Bereiche, in denen noch signifikante Wissenslücken bestehen, betreffen beispielsweise neue umweltrelevante Chemikalien und Produkte, z.B. synthetische Nanopartikel, die gleichzeitige Betrachtung von Umwandlungsprodukten der Chemikalien, die Langzeit-Persistenz und Bioverfügbarkeit von Boden- und Sediment-gebundenen Substanzen und der Aspekt der Wirkung von Chemikalienmischungen - eine Situation, die in der Umwelt regelmäßig anzutreffen ist.

Unter Federführung des Helmholtz-Zentrums für Umweltforschung - UFZ und dem Helmholtz-Forschungsprogramm Chemicals in the Environment (CITE) [2] wurde kürzlich eine Initiative gestartet, die die Belange der Ökotoxikologie und der Umweltchemie 
analysieren und koordinierte Forschungsprogramme zwischen verschiedenen Forschungsinstitutionen (Helmholtzzentren, Universitäten und Ressortforschung) anregen möchte. Dazu sollen gemeinsame Themen sowie strategische Fragen zu Schadstoffbezogenen Umweltwissenschaften auf nationaler Ebene diskutiert und abgestimmt werden. Damit wird eine Erhöhung der Sichtbarkeit der Themen in der deutschen Forschungsförderung angestrebt.

Bei der Jahrestagung der Society of Environmental Toxicology and Chemistry - German Language Branch (SETAC-GLB) 2009 in Weihenstephan fand eine Diskussion am Runden Tisch unter Moderation von Prof. Hollert (RWTH Aachen) zur Fördersituation in der ökotoxikologischen Forschung im deutschsprachigen Raum statt [3]. Prof. Braunbeck (Uni Heidelberg), Dr. Caspers (Currenta), Prof. Eisenträger (Umweltbundesamt), Dr. Hempel (DBU), Dr. Knacker (ECT) und Prof. Schäffer (RWTH Aachen und Sprecher der Initiative Umweltforschender Hochschulen, UFoH) konnten für die Diskussion gewonnen werden. Das Bundesministerium für Bildung und Forschung (BMBF) und die Deutsche Forschungsgemeinschaft (DFG) waren eingeladen, konnten aber aufgrund von terminlichen Kollisionen nicht teilnehmen. Nach einem Bericht über den Status Quo der Fördersituation wurden in einer ersten Diskussionsrunde die Stärken und Schwächen der Fördersituation der Ökotoxikologie besprochen. Es zeigte sich, dass trotz des großen Nachwuchsmangels und wichtiger Forschungsaufgaben in der Ökotoxikologie derzeit die großen öffentlichen Forschungsförderer (wie DFG, BMBF und DBU) nur wenige Forschungsprojekte in der Ökotoxikologie fördern. Diese verringerte Forschungsaktivität spiegele sich nach einer Literaturauswertung von Lammel und Mitautoren [4] bereits in verringerten internationalen Publikationsaktivitäten deutscher Autoren wider. Als großes Problem wurde gesehen, dass ökotoxikologische Projekte für das BMBF oft zu grundlagenorientiert, aber für die DFG $\mathrm{zu}$ anwendungsorientiert seien. Die DBU hat bisher keinen Schwerpunkt für die ökotoxikologische Forschung definiert. Im Gegensatz zu der zurückgegangenen Forschungsförderung durch die öffentlich Hand fördert die chemische Industrie derzeit zahlreiche Forschungsprojekte, was bei der Podiumsdiskussion aber durchaus auch kritisch gesehen wurde. Vielmehr dürfe sich die öffentliche Hand aufgrund der großen Relevanz ökotoxikologischer Fragestellerungen keineswegs aus der Forschungsförderung zurückziehen. Im weiteren Verlauf wurden wichtige Felder ökotoxikologischer Förderung identifiziert und die Frage besprochen, wie eine nachhaltige Förderung der Ökotoxikologie im deutschsprachigen Bereich gewährleistet werden kann.
Bei dem Runden Tisch zeigte sich, dass nur wenige belastbare Daten zur Fördersituation der Ökotoxikologie und der gesamten Schadstoffbezogenen Umweltwissenschaften vorliegen. Daher wurde die Notwendigkeit gesehen, eine belastbare Umfrage zu initiieren, die einen umfassenden und objektiven Überblick über die Forschungsföderung in Deutschland erarbeiten sollte.

\section{Ziel und Umsetzung:}

Um eine objektive Analyse über die Fördersituation der Ökotoxikologie und Umweltchemie in Deutschland zu erhalten, wurde eine anonyme Online-Befragung vom Institut für Umweltforschung der RWTH Aachen unter Mitarbeit des Institutes für Soziologie der RWTH Aachen ausgearbeitet und mittels der Befragungsplattform Survey Monkey technisch umgesetzt. Mit Unterstützung der Society of Environmental Toxicology (SETAC) - German Language Branch und der Gesellschaft Deutscher Chemiker (GDCh) - Fachgruppe für Umweltchemie und Ökotoxikologie wurde eine Einladung zur Teilnahme an der Befragung an alle Mitglieder der beiden maßgeblichen Verbände der Ökotoxikologie und Umweltchemie im deutschsprachigen Raum versendet. Nur leitende Mitarbeiter aus den Bereichen der Forschung, Behörden und Industrie sollten an der Befragung teilnehmen. Im „Ergänzenden Material“ (additional files 1 und 2) zu diesem Artikel sind weiterführende Informationen, die verwendeten Akronyme und alle Fragen dargestellt.

\section{Ergebnisse}

Hier soll nur eine Zusammenfassung der Ergebnisse der Befragung dargestellt werden. Der Original-Bericht aus der Survey Monkey-Umfrage und eine umfangreiche Auswertung seitens des Instituts für Soziologie der RWTH inklusive Kreuztabellen sind im „Ergänzenden Material“ (additional files 1 und 2) zu finden.

\section{Sozioökonomische Daten der teilnehmenden Wissenschaftler und Wissenschaftlerinnen}

Insgesamt haben 71 Wissenschaftler und Wissenschaftlerinnen aus verschiedenen Sparten (Abb. 1) in leitenden Positionen (Abb. 2) an der Befragung teilgenommen. Der Median des Drittmittelvolumens, welches von den Teilnehmern pro Jahr eingeworben wird, beträgt 100.000,-- $€$, der Mittelwert 245.000,-- $€$, der Maximalwert 1,7 Mio €. Durchschnittlich sind die Teilnehmer für 14 Mitarbeiter verantwortlich (Maximalwert: 70) und haben 24 ISI-gelistete Publikationen in den letzten 5 Jahren publiziert (Maximalwert 100). Die Leistungsdaten zeigen, dass die Teilnehmer als leistungsstark eingestuft werden können. Durchschnittlich verbringen die Befragten $5 \mathrm{~h}$ pro Woche mit dem Schreiben von Drittmittelanträgen (Min. 0,1 h, Max. 


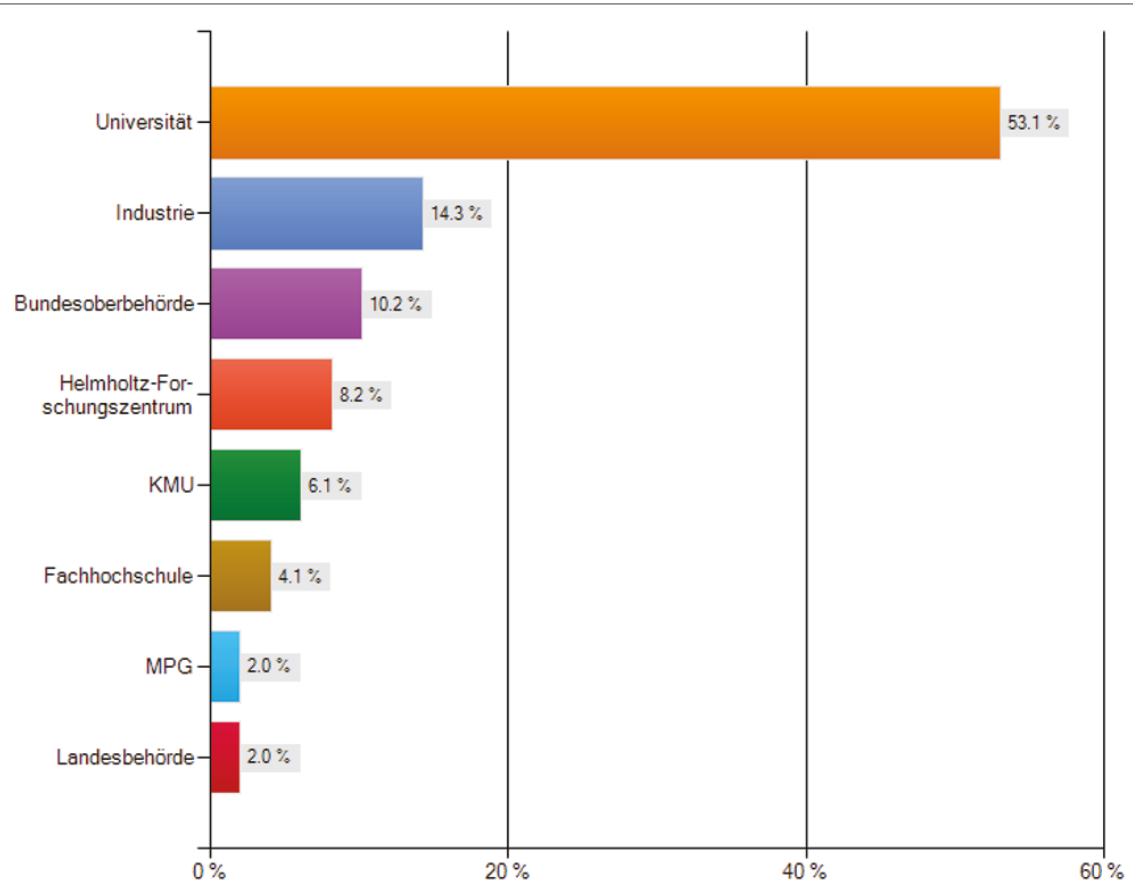

Abb. 1. Antworten auf die Frage:,/In welcher Sparte sind Sie beschäftigt?"

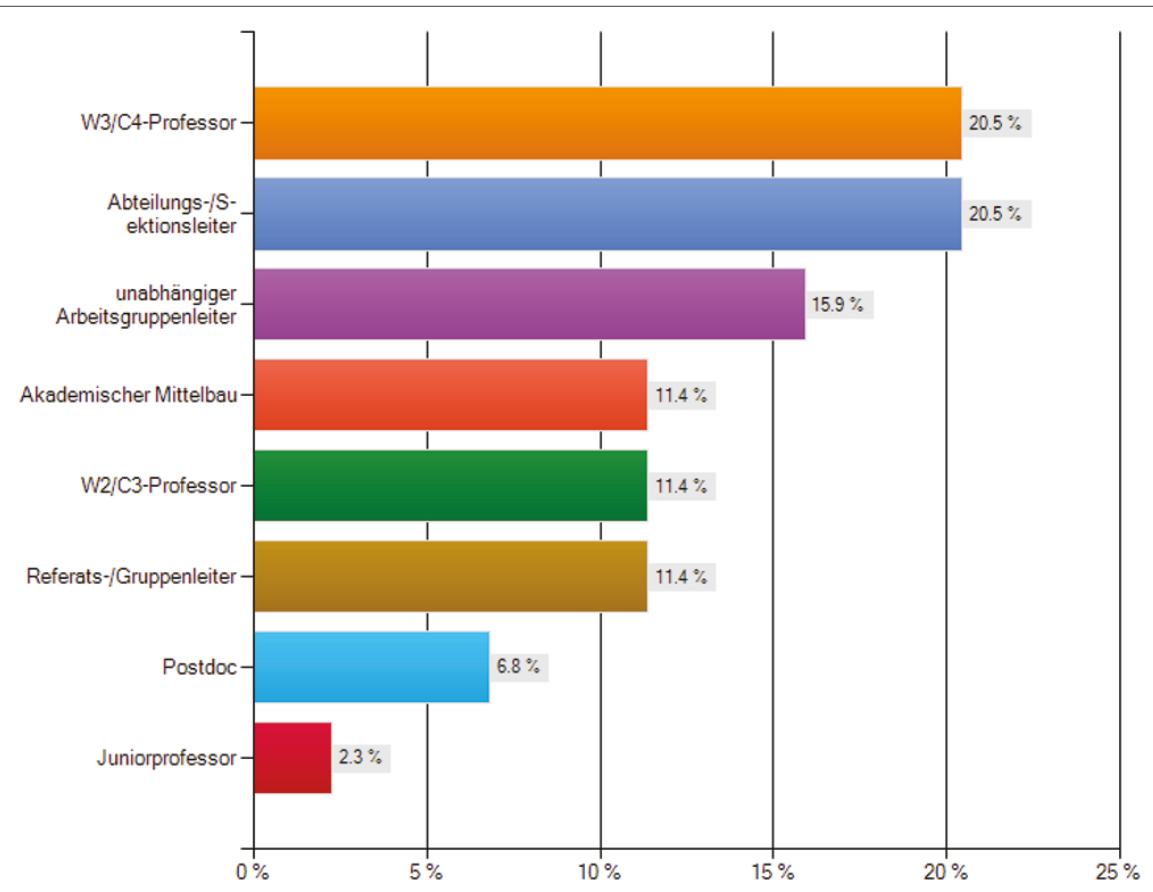

Abb. 2. Antworten auf die Frage: „Was ist Ihre Position?"

$16 \mathrm{~h}$ ), wobei die Erfolgsquote beim Einwerben von Drittmittel durchschnittlich $46 \%$ betrug. Die Befragten gaben an, dass Sie im Median 5 h pro Woche für das Schreiben von Publikationen aufwenden. Nur ein geringer Anteil der betreuten Doktorandinnen und
Doktoranden (unter 20\%) wurde durch DFG-Mittel finanziert, die verbleibenden wurden etwa $\mathrm{zu}$ gleichen Teil durch Promotionsstipendien (ohne DFG Graduiertenkollegs) und andere Drittmittelgeber (ohne DFG) finanziert. 


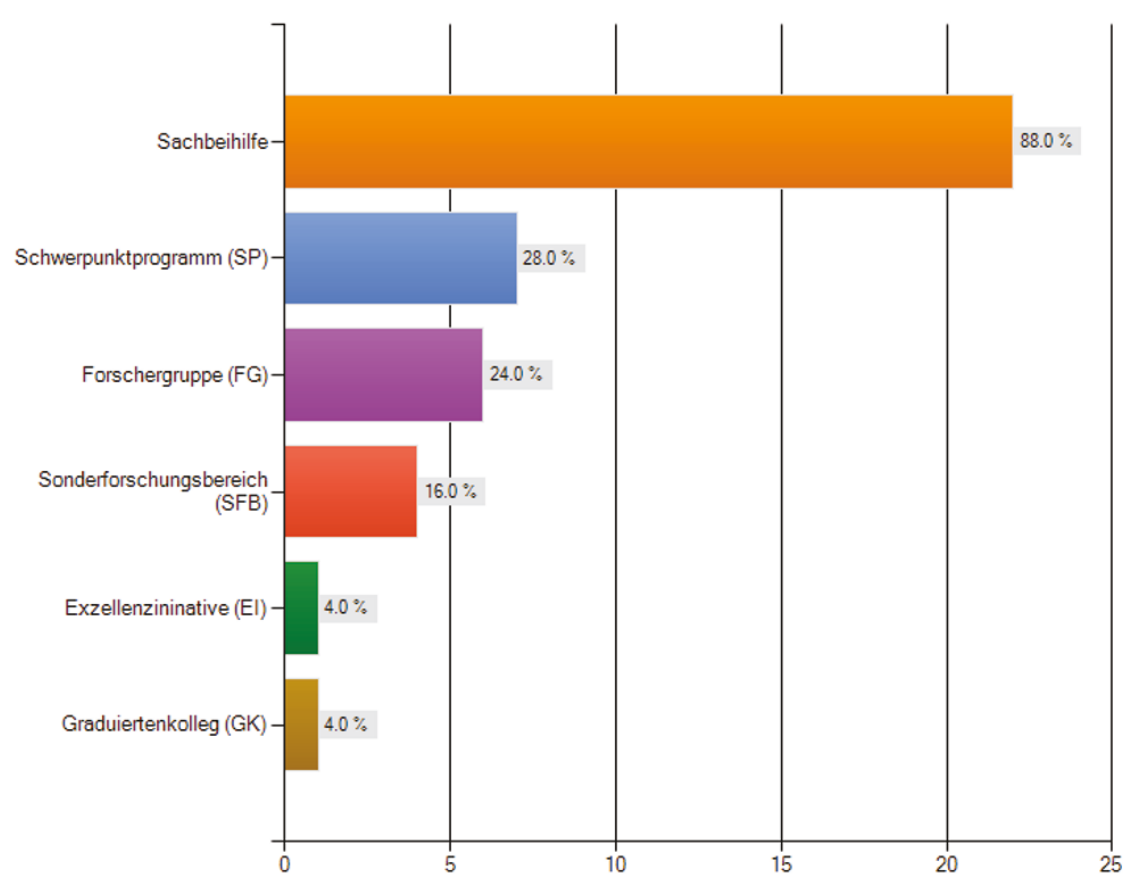

Abb. 3. Antworten auf die Frage: „Wenn Sie bei der DFG gefördert wurden: mit welchen Förderinstrumenten wurden Sie bei der DFG gefördert? (Mehrfachnennungen möglich)"

\section{Allgemeine Aussagen zur Förderung der Schadstoffbezogenen Umweltwissenschaften in Deutschland}

$62,3 \%$ der Befragten sind der Meinung, dass sich insgesamt die Förderung der Schadstoffbezogenen Umweltwissenschaften in den letzten Jahren verschlechtert oder deutlich verschlechtert hat. Der überwiegende Anteil der Befragten (60,9 \%) ist mit der Fördersituation durch Drittmittelgeber unzufrieden, nur $10,9 \%$ sind damit zufrieden. Auf die Frage „Ist die Forschungsförderung im europäischen Ausland insgesamt besser als in Deutschland?" antworteten $30 \%$ mit „ja“, $9 \%$ mit „nein“ und $61 \%$ mit „ich weiß nicht“.

\section{Förderung Schadstoffbezogener Umweltwissenschaften durch die DFG}

48,5\% der Befragten hatten bereits mindestens einen Antrag bei der DFG gestellt. Ein Drittel der Befragten gaben an, eine Förderung durch die DFG erhalten zu haben. In den überwiegenden Fällen erhielten die Wissenschaftler mit DFG-Förderung die Förderung aus Sachbeihilfen (88\%), gefolgt von Schwerpunktprogrammen, Forschergruppen und SFBs. Auffällig ist die nur geringe Förderung durch Graduiertenkollegien ( $\mathrm{n}=1$; $4 \%$ ) (vgl. Abb. 3). Bei den sonstigen Nennungen von DFG-Förderungen wurden auch ein Forschungsstipendium und ein Heisenberg-Stipendium genannt.
Die meisten Forscher mit DFG-Förderung gaben an, bisher eine Bewilligung erhalten zu haben. Nur etwa $20 \%$ der Befragten erhielten 3 oder mehr bewilligte DFGVorhaben, 10 \% der Befragten 5 oder mehr. Die Erfolgsquote war bei den Forschern mit bewilligten DFGProjekten in $56 \%$ aller Fälle besser als $33 \%$ und in $32 \%$ der Fälle besser als $12 \%$.

Abb. 4 zeigt, in welchen Fachbereichen die Anträge bei der DFG eingereicht wurden. Bei dieser Frage ist auffällig, dass die Anträge insgesamt sehr heterogen auf verschiedene Fachbereiche verteilt sind. Geowissenschaften, Wasserforschung und Chemie nehmen die ersten Ränge ein, vor den Kategorien „Anderer“, Biologie und Ökologie.

$64 \%$ sind mit der Förderung Schadstoffbezogener Umweltwissenschaften durch die DFG nicht zufrieden, nur $7 \%$ sind zufrieden (Abb 5.).

$29 \%$ der Befragten waren der Meinung, dass die Förderung von Schadstoffbezogener Umweltwissenschaften im europäischen Ausland (durch DFGäquivalente Förderer) besser als in Deutschland sei, $68 \%$ antworteten auf die Frage „weiß nicht" und $9 \%$ gaben an, dass die Förderung in Deutschland besser als im europäischen Ausland sei.

Die Befragten, die eine bessere Förderung durch DFGähnliche Förderungseinrichtungen im Ausland sahen, nannten folgende Argumente: 


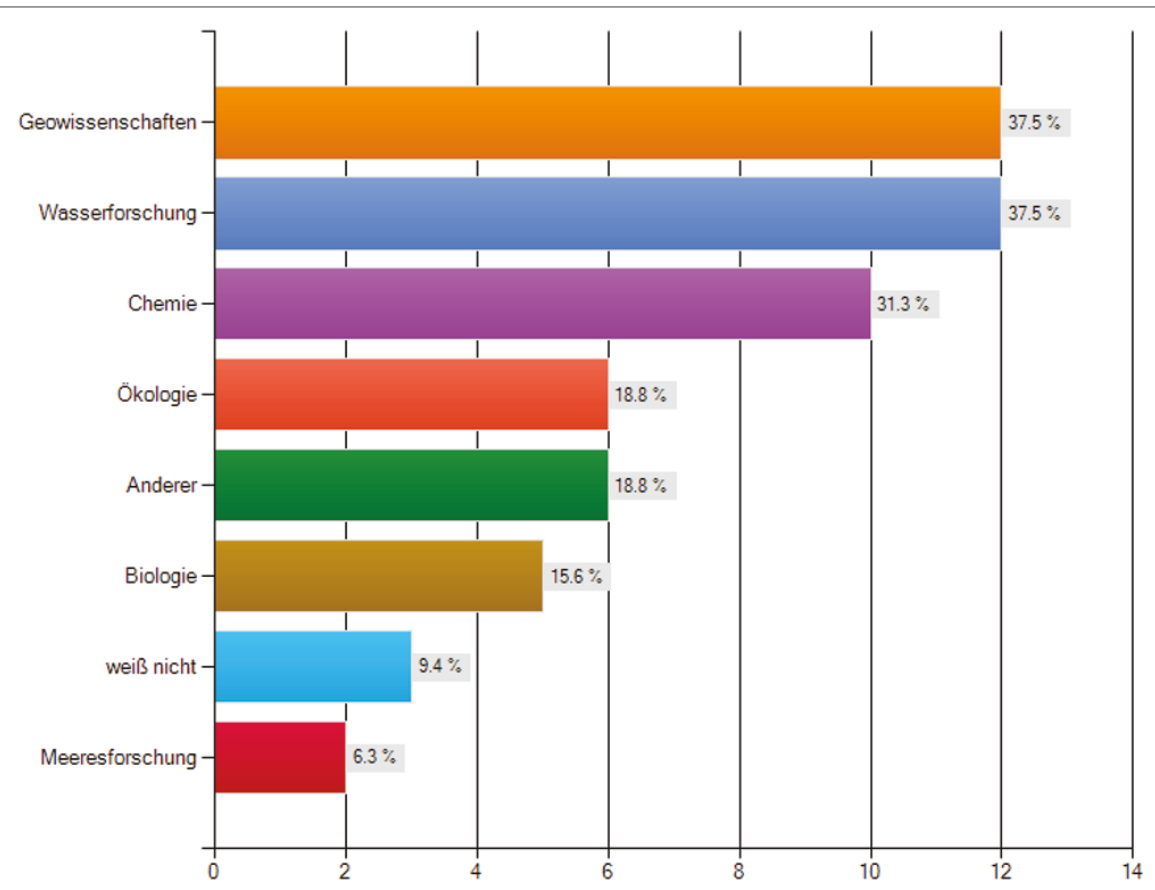

Abb. 4. Antworten auf die Frage: „In welchen DFG Fachbereichen wurden die Anträge eingereicht? (Mehrfachnennungen möglich)“

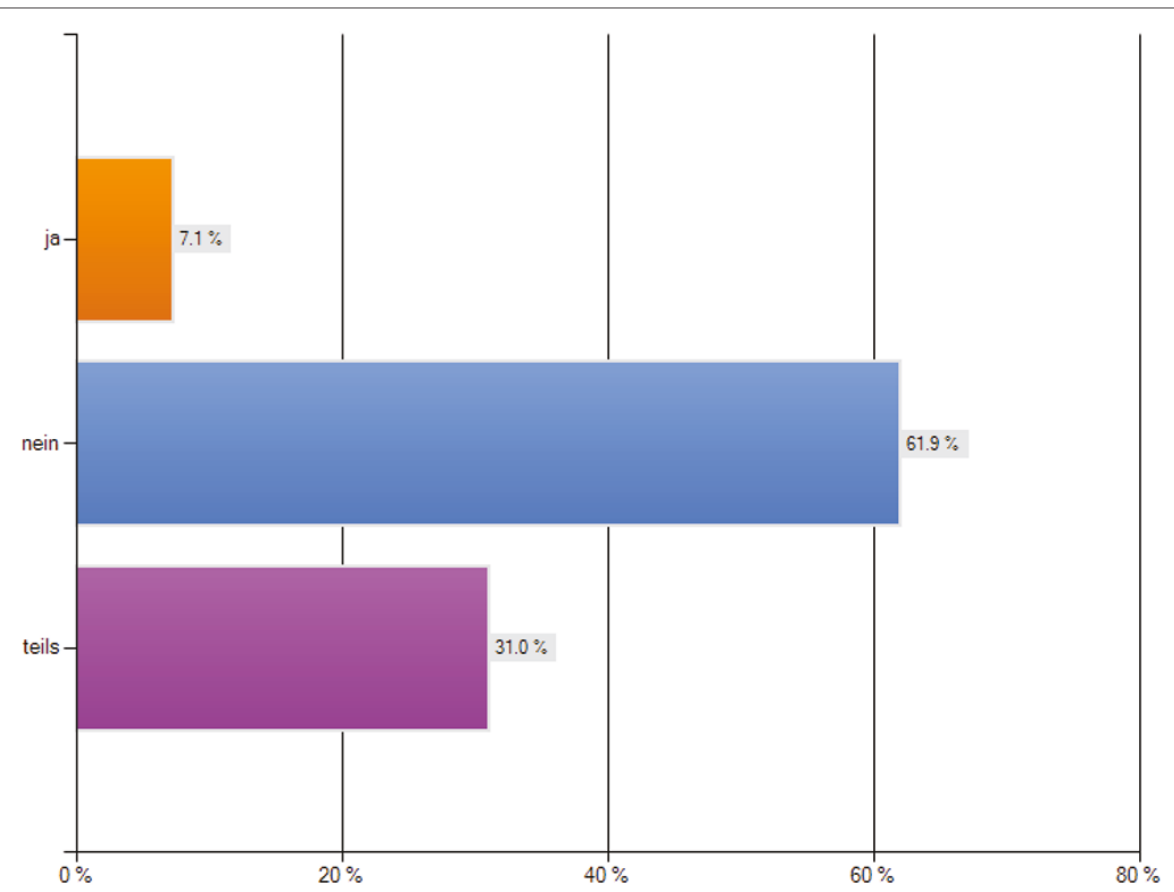

Abb. 5. Antworten auf die Frage: „Sind Sie mit der Förderung Schadstoffbezogener Umweltwissenschaften durch die DFG zufrieden?“

- Im Ausland gibt es oft eigene Referate

- Umweltwissenschaften werden mehr unterstützt (z.B. Niederlande, Schweiz)

- Bessere Vertretung der Umwelttechnik/Ökotoxikologie/ nachhaltigen Chemie in einschlägigen Gremien
- Höheres Interesse an substanzfokussierter Forschung

- Research Council in Groß-Britannien hat effektivere Verteilungs- und Bewertungsmechanismen

- Nationalfonds Schweiz (hohe Chancen auf dortige Förderung), Fonds zur Förderung der wissen- 
Die Anträge sind naturgemäß meist interdisziplinär, werden jedoch bei der Begutachtung oft monodisziplinär evaluiert. Soweit ich das erkennen kann, wird Interdisziplinarität zwar gerne eingefordert, kollidiert aber in der Praxis oft mit den noch monodisziplinär organisierten Fördertöpfen. Hier besteht dringender Handlungsbedarf.

Bessere Berücksichtigung interdisziplinärer Anträge. Obwohl offiziell gewünscht, scheint es mir je nach Gutachterauswahl und -expertise sowie Zusammensetzung des Fachkollegiums schwierig zu sein, diese auch bewilligt zu bekommen.

Eigener Bereich für die Ökotoxikologie

Einrichtung eines Förderungsschwerpunktes zu schadstoffbedingten Schäden für Umwelt und Gesundheit

Meine Erfahrung liegt einige Jahre zurück, aber damals hatte man das Gefühl, dass die Gutachter nichts vom Thema verstanden.

Ausweitung der antragsberechtigten Institutionen: konkret für privatwirtschaftliche Laboratorien u.ä.

Kompetentere Gutachter

Eigenständiges Sachgebiet mit Fachgutachtern

Wichtig wäre die Einrichtung eines Fachbereichs Umweltchemie und Ökotoxikologie bei der DFG, der die Schadstoffforschung in Deutschland mit expliziter Förderung interdisziplinärer Themen übernimmt.

Sinnvoll wäre die Schaffung weiterer Schwerpunktprogramme zu Themen wie nachhaltige Chemie, Ökotoxikologie, Stress Ecology und Risikobewertungsstrategien und Tools von Chemikalien, auch vor dem Hintergrund der Bedarfe und Weiterentwicklung von REACh.

Schaffung eines Fachkollegiums, Umweltchemie und Ökotoxikologie'

Eigenes Referat

Einbeziehung angewandter Forschung

Für dieses interdisziplinäre Thema sollte es einen eigenen Fachbereich oder Unterfachbereich geben.

Einrichtung eines eigenen, explizit interdisziplinären Fachbereichs, der gewährleistet, dass die Gutachter/innen einen entsprechend breiten Überblick über das Gebiet haben und nicht z.B. „reine" Molekularbiologen o.ä. sind

Überhaupt erst mal schaffen!

Bessere Anerkennung der Chemikalien-orientierten Forschung

Förderung von vernetzenden Projekten z.B. zu Datenbanken

Spezifische Ansprechpartner

Ökotoxikologie und Toxikologie als eigenständige Fächer etablieren

z.B. durch gezielte Ausschreibungen von Themen für Forschergruppen. Außerdem sind die Haupt-Gutachter oft zu stark in der Grundlagenforschung engagiert, so dass die sogenannte angewandte Grundlagenforschung (die wir betreiben) oft ohne reelle Chancen bleibt.

Intensivere / direkte Kommunikation mit den Antragstellenden. DFG sollte mehr dienstleisterisches Denken entwickeln.

Eigenes Umweltwissenschaftliches Ressort

Das Fachforum Chemie scheint oft anders als die Gutachter zu entscheiden. Das macht Entscheidungen oft nicht nachvollziehbar und macht die Gutachtertätigkeit überflüssig

Einrichtung eines speziellen Referats / Fachkollegiums für Chemikalien-bezogene Forschung - Höhere Gewichtung der anwendungsbezogenen und interdisziplinären Forschung - Stärkere Kommunikation zwischen Förderverantwortlichen und Forschenden - Stärkere Vernetzung der Förderinstitutionen (DFG, BMBF, DBU, BMU) zur Abstimmung von Förderprogrammen - Einführung von "Calls" wie auf EU-Ebene

Bessere Vertretung in den Fachgremien; Überwindung der disziplinären Grenzen (gerade davon lebt die Umweltforschung) und Abschaffung der Arroganz der älteren und „klassischen“ Fächer

Akzeptanz der Ökotoxikologie als Forschungsdisziplin!

Man sollte derartige Projekte nicht pauschal als „grundlagenfern“ oder „Zu anwendungsbezogen“ abstempeln und stattdessen das Verknüpfungspotential zwischen bspw. Chemie, Biologie und Geowissenschaften erkennen.

Durch Einrichtung eines fachübergreifenden Ressorts „Umweltwissenschaften"

Einrichtung einer Arbeitsgruppe einer Senatskommission. Einrichtung eines Referats "Chemikalien und Umwelt".

Es könnte z.B. ein Schwerpunktprogramm eingerichtet werden oder ein eigenes Referat. Ich habe den Eindruck, dass andere Fachwissenschaftler über Ökotoxikologie-Anträge entscheiden und hier die Anfordernisse des Faches zu wenig bekannt sind

*Die Antworten wurden redaktionell überarbeitet

schaftlichen Forschung Österreich, National Science Foundation USA

- Tschechien ist in der "Fächerstruktur" bei der Förderorganisation besser

- beste Förderungen in Brasilien und China

Auf die offene Frage, „Wie könnte die Förderung Schadstoffbezogener Umweltwissenschaften bei der DFG verbessert werden“, wurden die in Tab. 1 dargestellten Antworten gegeben. Auffällig ist, dass die Mehrheit der
Antworten die mangelnde Interdisziplinarität bei der DFG moniert oder die Einrichtung eines einschlägigen Fachkollegiums einfordert.

Förderung Schadstoffbezogener Umweltwissenschaften durch andere Förderinstitutionen

91,2 \% der Befragten gaben an, dass Sie bereits Drittmittelanträge bei anderen Förderinstitutionen gestellt haben, 83,6 \% haben bereits solche Anträge bewilligt 


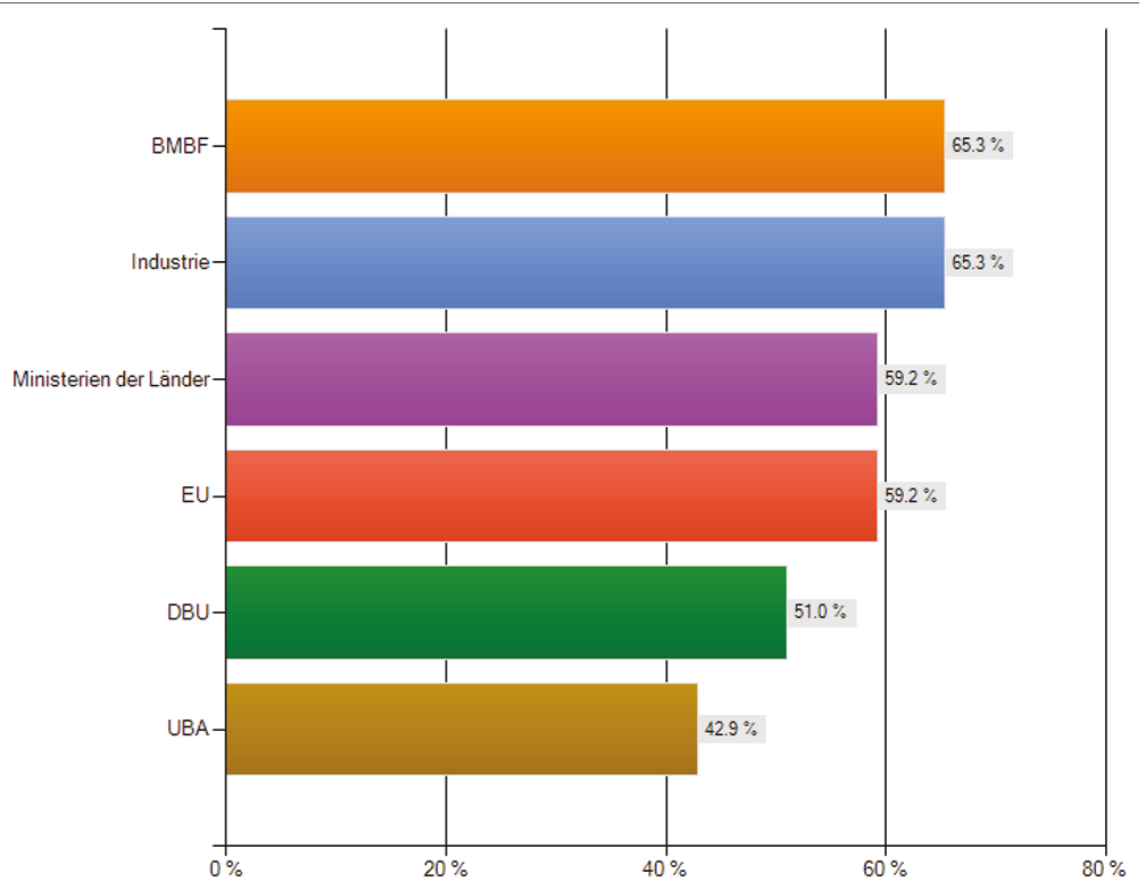

Abb. 6. Antwort auf die Frage: „Von welchen anderen Geldgebern haben Sie Förderung erhalten? (Mehrfachnennungen möglich)“

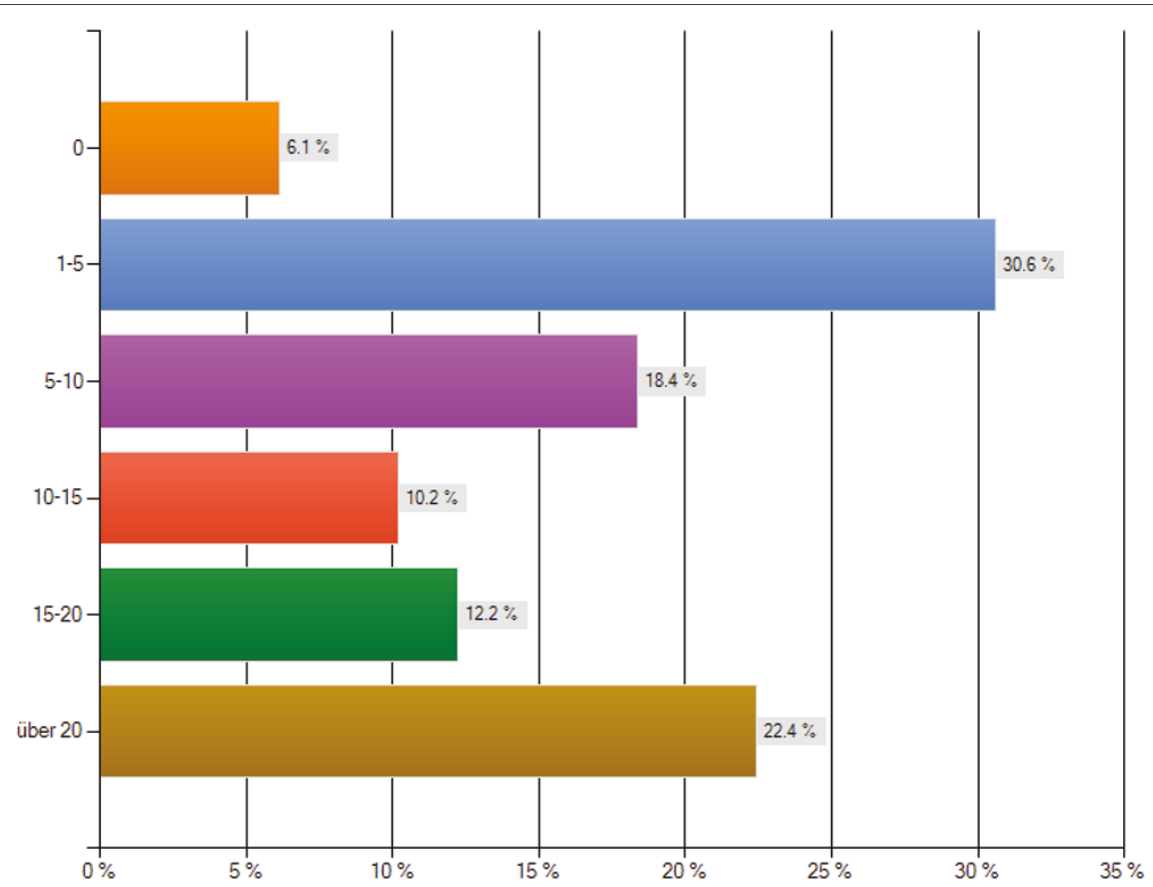

Abb. 7. Antwort auf die Frage:„Wie viele Drittmittelprojekte wurden Ihnen bisher insgesamt bewilligt?"

bekommen. Abb. 6 zeigt, bei welchen Geldgebern die Befragten bereits Förderung erhalten haben (Mehrfachnennungen waren möglich). Neben diesen Geldgebern wurden auch weitere genannt, wie etwa Universitätsinterne Förderungen, Stiftungen, VCI, CEFIC, AIF, BfR, DAAD und Behörden anderer EU-Staaten. Bei den
Befragten, die eine Förderung vom BMBF erhielten, wurden 81,5 \% vom Projektträger PTJ gefördert, 40,7 \% vom FZK-KIT und $37 \%$ vom DLR.

Abb. 7 zeigt, wie viele Drittmittelprojekte den Befragten bisher insgesamt bewilligt wurden. Dabei ist zu beachten, dass bei der Frage kein spezifischer Zeitraum 
Tab. 2. Antworten auf die Frage „Wie könnte die Förderung Schadstoffbezogener Umweltwissenschaften insgesamt verbessert werden"

Eigenes Ressort bei der DFG

Verzicht auf rein programmorientierte Förderung wie derzeit beim BMBF; Definition von entsprechenden Forschungs-Schwerpunkten

Eigene Bereiche für die Ökotoxikologie wären gut

Mehr öffentliche Aufmerksamkeit für das Thema Umwelt- und Gesundheitsschäden durch Chemikalien

Einrichtung von Lehrstühlen, Grundausstattung der Arbeitsgruppen

Realistische Einschätzung der nötigen Ressourcen: Verhältnis Arbeit zu Förderung wird deutlich schlechter

Sichtbarkeit als Thema bei großen Geldgebern

Entsprechende Fachbereiche bzw. Projektträger mit ausreichender Ausstattung für Chemikalien-bezogene Forschung

Bedarf aufzeigen, Neuerungen fördern, Finanzierung durch Industrie

Mehr Fördermittel zur Erforschung von Expositionsfragen

Erhöhung des Anteils längerfristiger Projekte (3-5 Jahre; in der letzten Zeit hat der Anteil der sehr kurzfristigen Projekte stark zugenommen). Erhöhung der Anzahl von Projekten, die sich hauptsächlich mit den Auswirkungen von Chemikalien befassen.

Vereinfachte Antragstellung, schneller aktuelle Themen u. Gefahrstoffe aufgreifen, Einbeziehen der Industrie

Es müsste mehr in den öffentlichen Fokus gebracht werden, wie wichtig diese Forschung vor allem auch aus gesellschaftlicher und wirtschaftlicher (VWL und BWL) Sicht ist.

Fach ist nicht anerkannt: Grundlagencharakter wird ignoriert

Bessere Abstimmung zwischen den Förderinstitutionen

Integrativer, kooperativer, Einbeziehung verschiedener Disziplinen. Aber dann auch harte Kontrollen des Erfolgs der Kooperation

Bezug in aktuellen Ausschreibungen und Förderschwerpunkte. Eine Biodiversitätsdiskussion ohne die Wirkung von Chemikalien einzubeziehen ist auf dieser Welt nicht realistisch und fahrlässig.

Mehr zweckfreie Grundlagenforschung zur fundierten Theorieentwicklung des Forschungsgebiets

UBA hat heute für reine Forschung etwa $10 \%$ des Budgets von 1990 - das sagt alles

Verbindung der Forschung mit anderen relevanten Gebieten wie Klimaforschung, Biodiversität,...

Mehr Kontext-bezogene Anträge, mehr Anträge mit direktem Nutzen, Umsetzbarkeit, Relevanz für die Gemeinschaft

Mehr Projekte zu aktuellen Themen, sowie Langzeitprojekte zu übergeordneten Themen

Es müssten mehr dauerhafte Perspektiven für den sogenannten akademischen Mittelbau geschaffen werden, die Verluste in der Forschung an sehr guten Leuten, die dann aber ins Ausland gehen oder in die Industrie ist extrem hoch und wird langfristig für Deutschland ein Problem darstellen. Ich selbst bin seit ca. einem 3/4 Jahr im Ausland.

Durch gezielte Problem-Identifikation und Ausschreibung nach Konsultation von möglichst vielen Beteiligten (wo sind die Fragestellungen auch von öffentlicher Seite)

Förderer müssen stärker kundenorientiert werden.

Entbürokratisierung

Stärkere Vernetzung und gemeinsame Artikulation der Forschenden (wie UFOH-Initiative) /Schwerpunktsetzungen beim Forschungsbedarf von Seiten der Politik und Behörden/ Stärkere Einbindung der öffentlichen Forschung bei der Bewertung, Zulassung und Regulation von Chemikalien

Förderung von Transdisziplinarität nicht nur auf dem Papier und mit blumigen Worten, sondern de facto

Offenere Umgangsweise der Förderinstitutionen mit dem Thema

Ist auf zu viele Ressorts verteilt:Wasserforschung, Atmosphärenforschung, Bodenforschung, Geowissenschaften, wobei sie jeweils nur randständig bleibt. Eigenes Ressort "Umweltforschung" unter Einbeziehung aller Kompartimente wäre hilfreich.

s.o. (DFG)

Fast alle Förderer haben sich stark zurückgezogen aus der ökotoxikologischen Förderung

vorgegeben war und durch die Struktur der Befragten hauptsächlich Personen in Leitungsfunktionen mit z.T. jahrzehntelanger Forschungserfahrung Antworten gaben. Dennoch zeigt die Abbildung, dass die Mehrzahl der Teilnehmenden sehr erfolgreich Drittmittel eingeworben hat. Dies steht in krasser Diskrepanz zur Situation bei der DFG (siehe Abb. 3 und zugehöriger Text), die sowohl bei der Antragstellung (48,5\% vs. 91,2\%) und Förderung (33\% vs. 83,6\%) deutlich unter den übrigen Mittelgebern liegt.

Auf die offene Frage, „Wie könnte die Förderung Schadstoffbezogener Umweltwissenschaften insgesamt verbessert werden" wurden die in Tab. 2 dargestellten Antworten gegeben, die aufgrund ihrer Bedeutung hier entsprechend den Ergebnissen in Tab.1 in Gänze dargelegt werden. Die vielfältigen Anregungen verdeutlichen das nicht umgesetzte Potenzial der ökotoxikologischen Forschung und zahlreiche wichtige, derzeit nicht angemessen berücksichtigte Fragen. Die angesprochenen Aspekte spannen einen weiten Bogen von der Grundlagenforschung über die Ausbildung bis hin zur Öffentlichkeitsarbeit und zu strukturellen Erwägungen der Forschungsförderung.

\section{Diskussion und Schlussfolgerungen:}

Zusammenfassend verdeutlicht die Befragung, dass die Fördersituation der Ökotoxikologie und Umweltchemie 
in Deutschland insgesamt als steigerungsbedürftig, bei der DFG jedoch als problematisch zu bewerten ist. Insbesondere die Antworten zu Verbesserungsmöglichkeiten der Forschungsförderung sollten als Grundlage für einen offenen Dialog der Chemikalien-bezogenen Umweltforschung mit den Drittmittelgebern DFG, BMBF und DBU und den entsprechenden Institutionen in der Schweiz und in Österreich genutzt werden. Initiativen, große Verbundprojekte zu diesem Themenbereich mit einer langfristigen Forschungsförderungsperspektive vorzubereiten, sollten unterstützt werden, z.B. durch Finanzierung von Rundgesprächen und Workshops.

Aus den Antworten bezüglich der Verbesserungsmöglichkeiten der Förderung Schadstoffbezogener Umweltwissenschaften bei der DFG (siehe Tab.1) können folgende Empfehlungen für diese Förderinstitution abgeleitet werden:

- Wiederholt wurden interdisziplinäre Fördertöpfe angeregt,

- ebenso einen eigenen Ansprechpartner, ein eigenes Referat bis hin zu einem eigenen Resort zu diesem Forschungsfeld.

- Es sollte ein DFG-Fachkollegium zu Schadstoffbezogenen Umweltwissenschaften oder eine Arbeitsgruppe zur DFG-Senatskommission eingerichtet werden. Dabei ist sicherzustellen, dass die Angehörigen eine breite und vor allem interdisziplinäre Expertise vorweisen können

- Zur Gewährleistung einer theoriegeleiteten Weiterentwicklung des Fachgebiets sind langfristige, koordinierte Programme (gezielte Ausschreibung sowie die Schaffung eines Schwerpunktprogramm) erforderlich Aus dem Antwortverhalten zu den generellen Verbesserungsvorschlägen zur Förderung einer Chemikalien-bezogenen Forschung (siehe Tab.2) können folgende drei Empfehlungen an die Akteure in diesem Forschungsfeld selbst abgeleitet werden:

- Die Öffentlichkeitsarbeit muss intensiviert werden.

- Ebenso wurde mehrfach der Aspekt der Sichtbarkeitserhöhung der Chemikalien-bezogenen Forschung an den Universitäten und Forschungseinrichtungen (zum Beispiel durch eine entsprechende Berufungspolitik, Schaffung von Studienmodulen bzw. Studiengängen etc.) und in Richtung der klassischen naturwissenschaftlichen Disziplinen genannt.
- Ferner wurde der Bedarf einer längerfristig angelegten Grundlagenforschung angemahnt, um das Forschungsfeld theoretisch zu fundieren.

\section{Additional files}

\section{Additional file 1. Fragebogenaktion zur Förderung chemikalienbezogener Forschung.}

Additional file 2. Einladungs Email.

Author details

'Department of Ecosystem Analysis, Institute for Environmental Research (Biology V), RWTH Aachen University, Worringerweg 1, 52074 Aachen, Germany. ${ }^{2}$ UFT, Department of General and Theoretical Ecology, University of Bremen, Leobener Str., 28359 Bremen, Germany. ${ }^{3}$ Sociology of Technology and Organization, Institute of Sociology, RWTH Aachen University, Eilfschornsteinstraße 7, 52062 Aachen, Germany. ${ }^{4}$ CITE (Chemicals In The Environment), Department Bioanalytical Ecotoxicology, Helmholtz Centre for Environmental Research - UFZ, Permoserstraße 15 / 04318 Leipzig, Germany. ${ }^{5}$ nstitute for Environmental System Research, University of Osnabrück, Barbarastr. 12, 49069 Osnabrück, Germany. ${ }^{6}$ Department Aquatic Ecotoxicology, Institute for Ecology, Evolution and Diversity, Goethe University Frankfurt am Main, Siesmayerstrasse 70, 60054 Frankfurt am Main, Germany. ${ }^{7}$ Chair for Environmental Biology and Chemodynamics, Institute for Environmental Research (Biology V), RWTH Aachen University, Worringerweg 1, 52074 Aachen, Germany. ${ }^{8}$ Safety and Environmental Technology Group, ETH Zürich, HCI G 127, Wolfgang-Pauli-Str. 10, 8093 Zürich, Switzerland.

Received: 29 June 2011 Accepted: 06 July 2011

Published: 06 July 2011.

Literatur

1. Schaeffer A, Hollert H, Ratte HT, Roß-Nickoll M, Filser J, Matthies M, Oehlmann J, Scheringer M, Schulz R, Seitz A: An indispensable asset at risk: merits and needs of chemicals-related environmental sciences. Environ Sci Pollut Res 2009, 16:410-413.

2. Altenburger R, Kühnel D, Bittens M, Daus B, BrackW, Centler F, Harms H, Thullner M, Wick LY, Goss KU, Kopinke FD, MacKenzie K, Miltner A, Liess M, Wennrich R, Berns AE, Burauel P: Chemicals in the environment (CITE): Report of the first annual conference of the Helmholtz research topic CITE. Umweltwiss Schadst Forsch 2010, 22:502-506.

3. Dawo U, Hollert H, Ebke KP, Huber W: 14. SETAC GLB Jahrestagung an der TU München in Weihenstephan. Umweltwiss Schadst Forsch 2010, 22:162-164.

4. Lammel G, Scheringer M, Fischer K: Umweltchemie und Ökotoxikologie in Deutschland - Entwicklungstrends des Faches gemessen an der Publikationsaktivität. Umweltwiss Schadst Forsch 2009, 21:407-411.

doi:10.1186/2190-4715-23-24

Cite this article as: Hollert $H$, et al.: Financial Research Support for Ecotoxicology and Environmental Chemistry in Germany - Results of an Online Survey. Environmental Sciences Europe 2011, 23:24. 